\title{
Decay rates at infinity for solutions to periodic Schrödinger equations
}

\author{
Daniel M. Elton \\ Department of Mathematics and Statistics, Fylde College, Lancaster \\ University, Lancaster LA1 4YF, UK
}

(MS received Received date; Accepted date)

\begin{abstract}
We consider the equation $\Delta u=V u$ in the half-space $\mathbb{R}_{+}^{d}, d \geq 2$ where $V$ has certain periodicity properties. In particular we show that such equations cannot have non-trivial superexponentially decaying solutions. As an application this leads to a new proof for the absolute continuity of the spectrum of particular periodic Schrödinger operators. The equation $\Delta u=V u$ is studied as part of a broader class of elliptic evolution equations.
\end{abstract}

\section{Introduction}

We are interested in the possible decay rate of (distributional) solutions to the equation

$$
(-\Delta+V) u=E u
$$

where $\Delta$ is the Laplace operator on $\mathbb{R}^{d}, V$ is a measurable function and $E$ is a constant. Landis (see [6]) asked if the boundedness of $V$ is sufficient to exclude superexponentially decaying solutions. More precisely, suppose $V$ is bounded and $u$ solves (1.1) in the exterior region $\Omega_{R}=\left\{x \in \mathbb{R}^{d}:|x|>R\right\}, R>0$, while $\mathrm{e}^{\lambda|x|} u$ is bounded on $\Omega_{R}$ for all $\lambda>0$; does it follow that $u \equiv 0$ on $\Omega_{R}$ ?

Viewing $E$ as a spectral parameter (1.1) becomes the spectral equation for the Schrödinger operator with potential $V$. In this context Simon ([12]) posed a related question about superexponentially decaying solutions; in particular, if $V$ is such that $-\Delta+V$ defines a self-adjoint operator with a non-compact resolvent does any non-trivial solution of (1.1) satisfy $\mathrm{e}^{\lambda|x|} u \notin L^{2}$ for some $\lambda>0$ ? Note that, $V$ must be real-valued for $-\Delta+V$ to be self-adjoint, while $-\Delta+V$ has a non-compact resolvent for any bounded $V$.

If one considers complex-valued $V$ the answer to Landis' question is negative. In particular, given $\varepsilon \in[0,1 / 2)$ there exists a continuous complex-valued $V$ on $\mathbb{R}^{2}$ and non-trivial $u \in C^{2}\left(\mathbb{R}^{2}\right)$ with $V(x)=O\left(|x|^{-\varepsilon}\right)$ as $|x| \rightarrow \infty, \Delta u=V u$ on $\mathbb{R}^{2}$, and $\mathrm{e}^{\lambda|x|^{(4-2 \varepsilon) / 3}} u \in L^{\infty}$ for some $\lambda>0$; see [9] for the case $\varepsilon=0$ and [2] for the generalisation to $\varepsilon>0$.

On the other hand Landis' question is known to have a positive answer when $d=1$ (essentially a classical result for ordinary differential equations), when $d=2$, $E=0$ and $V \geq 0([5])$, and for any $E \in \mathbb{R}$ and $V$ with $V(x)=O\left(|x|^{-1 / 2}\right)$ as $|x| \rightarrow$ $\infty([3,8])$. For $E \in \mathbb{R}$ and bounded real-valued $V$, superexponentially decaying solutions of (1.1) can also be excluded under some conditions which stabilise $V(x)$ 
for large $x$; in particular this holds when (the distributional derivative) $(x . \nabla) V$ is also bounded $([1,3])$. A complete answer to Landis' question for real-valued potentials, or to the more general question of Simon, remains unknown.

In the present work we consider functions $V$ which are periodic transverse to a given direction. This naturally favours working on a half-space; since $\Omega_{R}$ includes a translated copy of any half-space our results will also apply to exterior regions. Let $\mathbb{R}_{+}=(0,+\infty)$ and $\mathbb{R}_{+}^{d}=\mathbb{R}^{d-1} \times \mathbb{R}_{+}$. For $x \in \mathbb{R}_{+}^{d}$ we will use the notation $x=(\widetilde{x}, t)$ where $\widetilde{x}=\left(x_{1}, \ldots, x_{d-1}\right) \in \mathbb{R}^{d-1}$ and $t=x_{d}>0$. Also set $\langle\widetilde{x}\rangle=\left(1+|\widetilde{x}|^{2}\right)^{1 / 2}$. We obtain the following.

Theorem 1.1. Suppose $V \in L^{\infty}\left(\mathbb{R}_{+}^{d}\right)$ is periodic with respect to a lattice $\Gamma \subset \mathbb{R}^{d-1}$ and $E \in \mathbb{R}$. Let $u \in L_{\text {loc }}^{2}$ be a (distributional) solution of (1.1) on $\mathbb{R}_{+}^{d}$ which satisfies

$$
\int_{\mathbb{R}_{+}^{d}}\langle\widetilde{x}\rangle^{2 \kappa} \mathrm{e}^{2 \lambda t}|u(x)|^{2} \mathrm{~d} \widetilde{x} \mathrm{~d} t<+\infty
$$

for all $\lambda>0$ and some $\kappa$. Suppose we also have (at least) one of the following:

(i) $d=2$ and $\kappa \geq 0$.

(ii) $d=3, \Gamma$ is rational and $\kappa>1$.

(iii) $d \geq 2$, $\Gamma$ is rational, $\kappa>(d-1) / 2$ and $\|V(\cdot, t)\|_{L^{\infty}\left(\mathbb{R}^{d-1}\right)} \rightarrow 0$ as $t \rightarrow+\infty$.

Then $u \equiv 0$ on $\mathbb{R}_{+}^{d}$.

By periodicity of $V$ with respect to $\Gamma$ we mean $V(\widetilde{x}+l, t)=V(\widetilde{x}, t)$ for any $(\widetilde{x}, t) \in \mathbb{R}_{+}^{d}$ and $l \in \Gamma$.

Remark. Note that $V$ is allowed to be complex-valued. In cases (i) and (ii) we can absorb $E$ into $V$; it follows that we can allow complex $E$ in these cases. Also note that the conditions on $V$ are satisfied by any potential which is periodic with respect to a lattice on $\mathbb{R}^{d}$, provided this lattice has a rational rank $d-1$ sublattice.

Remark. Exponential decay (namely (1.2) for some $\lambda>0$ ) is not sufficient. For example,

$$
u(x)=\frac{e^{i\left(x_{1}+i x_{2}\right)}}{\left(x_{1}+i x_{2}\right)+i}
$$

defines a harmonic function on $\mathbb{R}_{+}^{2}$ (so $(-\Delta+V) u=0$ with $\left.V=0\right)$ while $e^{\lambda x_{2}} u \in$ $L^{2}\left(\mathbb{R}_{+}^{2}\right)$ for any $\lambda<1$; however $u \not \equiv 0$.

Theorem 1.1 and related results on the non-existence of solutions with certain types of decay can be viewed as unique continuation theorems at infinity for (1.1). The implied lower bounds on the decay rate (possibly in a more quantitative form) have applications to spectral questions for Schrödinger operators (such as the exclusion of embedded eigenvalues; see [4] for example). For periodic potentials an important link was established in [7, theorem 4.1.5]; in particular, if $V \in L^{\infty}\left(\mathbb{R}^{d}\right)$ is periodic (with respect to a lattice on $\mathbb{R}^{d}$ ) then the self-adjoint operator $-\Delta+V$ has an eigenvalue iff (1.1) has a superexponentially decaying solution. As a corollary of theorem 1.1 we thus obtain a new proof for the following particular case of a well known result of Thomas ([13]). 
Theorem 1.2. Let $d=2$ or 3 and suppose $V \in L^{\infty}\left(\mathbb{R}^{d}\right)$ is real-valued and periodic with respect to a lattice on $\mathbb{R}^{d}$ which contains a rational rank $d-1$ sublattice. Then the spectrum of the self-adjoint operator $-\Delta+V$ contains no eigenvalues.

Existing proofs of this result and its many generalisations make use of Bloch (or Floquet) analysis and the analytic extension of the resulting operators into complex values of the quasi-momentum.

Theorem 1.1 is obtained as a special case of a more general result which we now describe. Let $A$ be a lower semi-bounded self-adjoint operator on a Hilbert space $X$. For $j=0,1,2$ set $X_{j}=\operatorname{Dom}\left(|A|^{j / 2}\right) \subseteq X$, so $X_{2}=\operatorname{Dom}(A), X_{1}$ is the form domain of $A$ and $X_{0}=X$. For $j=1,2$ we can make $X_{j}$ into a Hilbert space using the isomorphism $|A|^{j / 2}+I: X_{j} \rightarrow X$.

Let $\nabla_{t}$ denote differentiation with respect to $t$. We want to consider the operator $\nabla_{t}^{2}-A$ which maps $\mathcal{X}_{\mathrm{loc}}^{2} \rightarrow \mathcal{X}_{\mathrm{loc}}^{0}$ where

$$
\mathcal{X}_{\mathrm{loc}}^{2}=L_{\mathrm{loc}}^{2}\left(\mathbb{R}_{+}, X_{2}\right) \cap H_{\mathrm{loc}}^{1}\left(\mathbb{R}_{+}, X_{1}\right) \cap H_{\mathrm{loc}}^{2}\left(\mathbb{R}_{+}, X\right) \quad \text { and } \quad \mathcal{X}_{\mathrm{loc}}^{0}=L_{\mathrm{loc}}^{2}\left(\mathbb{R}_{+}, X\right) .
$$

We are interested in the possible decay rate of functions $\phi \in \mathcal{X}_{\text {loc }}^{2}$ which satisfy $\left(\nabla_{t}^{2}-A\right) \phi=B_{t} \phi$ where $t \mapsto B_{t}$ is a uniformly bounded family of operators on $X$. At this general level we obtain the following.

Theorem 1.3. Let $\phi \in \mathcal{X}_{\mathrm{loc}}^{2}$ and suppose $\left\|\left(\nabla_{t}^{2}-A\right) \phi(t)\right\|_{X} \leq \beta\|\phi(t)\|_{X}$ for some $\beta>0$ and all $t>0$. If $\phi$ satisfies

$$
\int_{0}^{\infty} \mathrm{e}^{2 \lambda t^{4 / 3}}\|\phi(t)\|_{X}^{2} \mathrm{~d} t<+\infty
$$

for all $\lambda>0$, then we must have $\phi \equiv 0$.

This result extends [9, theorem 1]; indeed we can recover the latter by taking $A$ to be minus the Laplacian on $\mathbb{R}^{d-1}$. Furthermore the example constructed in $[9, \S 2]$ shows the decay rate limits given by theorem 1.3 cannot be improved in general. To exclude any non-trivial solutions with superexponential decay as $t \rightarrow+\infty$ we impose further conditions on the gaps in the spectrum of $A$, possibly in conjunction with some form of decay for $B_{t}$.

Theorem 1.4. Let $\phi \in \mathcal{X}_{\mathrm{loc}}^{2}$ and suppose $\left\|\left(\nabla_{t}^{2}-A\right) \phi(t)\right\|_{X} \leq b(t)\|\phi(t)\|_{X}$ for some $b \in L^{\infty}\left(\mathbb{R}_{+}\right)$. Further suppose one of the following is satisfied:

(i) $\operatorname{spec}(A)$ contains arbitrarily large positive gaps.

(ii) there exists $\delta>0$ such that $\mathbb{R}_{+} \backslash \operatorname{spec}(A)$ contains infinitely many disjoint intervals of length $\delta$, and $b(t) \rightarrow 0$ as $t \rightarrow+\infty$.

If $\phi$ satisfies

$$
\int_{0}^{\infty} \mathrm{e}^{2 \lambda t}\|\phi(t)\|_{X}^{2} \mathrm{~d} t<+\infty
$$

for all $\lambda>0$, then we must have $\phi \equiv 0$. 
The conditions on $A$ are satisfied by a number of standard operators. For example, condition (i) holds if $A$ is minus the Laplace-Beltrami operator on the $n$-sphere $\mathbb{S}^{n}$ for $n \geq 1$, or any positive elliptic pseudo-differential operator of order $m$ on a closed $n$-dimensional manifold provided $m>n$; in the latter case the spectral part of condition (ii) is met when $m=n$. In order to deduce theorem 1.1 we need to consider the Laplacian on $(d-1)$-dimensional tori; the rationality assumption is then used to establish the existence of arbitrarily large positive gaps when $d=3$, or infinitely many gaps of a uniform size for arbitrary $d$ (see proposition 2.2 below). It is not known if arbitrarily large positive gaps exist for all 2-dimensional tori.

We can consider $\nabla_{t}^{2}-A$ as an 'elliptic evolution operator'. The corresponding parabolic and hyperbolic evolution operators, $\nabla_{t}+A$ and $\nabla_{t}^{2}+A$ respectively, were considered in [9] where results in the spirit of theorems 1.3 and 1.4 were obtained.

Theorems 1.3 and 1.4 are obtained from Carleman type estimates (see propositions 3.1 and 3.2 respectively) using standard arguments; these are presented in $\S 3$, with the proofs of the Carleman estimates being given in $\S 4$. In $\S 2$ we deduce theorem 1.1 from theorem 1.4 and consideration of gaps in the spectra of Laplace type operators on tori.

\section{Periodic result}

Let $\Gamma \subset \mathbb{R}^{d-1}$ be a lattice with $\Gamma^{\dagger} \subset \mathbb{R}^{d-1}$ denoting the dual lattice. Also let $\mathcal{O}$ and $\mathcal{O}^{\dagger}$ denote unit cells of $\Gamma$ and $\Gamma^{\dagger}$ respectively. Set $\mathbb{T}=\mathbb{R}^{d-1} / \Gamma$, the $(d-1)$ dimensional torus corresponding to $\Gamma$. For each $t \in \mathbb{R}_{+}$the function $\widetilde{x} \mapsto V(\widetilde{x}, t)$ is $\Gamma$-periodic so can be viewed as a function $V_{t} \in L^{\infty}(\mathbb{T})$; the mapping $t \mapsto V_{t}$ is uniformly bounded in $t$. We will apply a Bloch-Floquet decomposition to $-\Delta-E$ (see [11]); this leads to a family of lower semi-bounded self-adjoint elliptic operators on $\mathbb{T}$ defined by

$$
H_{\theta}=\left(-i \nabla_{\widetilde{x}}+\theta\right)^{2}-E
$$

for $\theta \in \mathcal{O}^{\dagger}$. The operator $\nabla_{t}^{2}-H_{\theta}$ maps $\mathcal{X}_{\text {loc }}^{2} \rightarrow \mathcal{X}_{\text {loc }}^{0}$ where

$$
\mathcal{X}_{\mathrm{loc}}^{2}=\bigcap_{j=0,1,2} H_{\mathrm{loc}}^{j}\left((1, \infty), H^{2-j}(\mathbb{T})\right) \quad \text { and } \quad \mathcal{X}_{\mathrm{loc}}^{0}=L_{\mathrm{loc}}^{2}\left((1, \infty), L^{2}(\mathbb{T})\right) .
$$

The Bloch-Floquet decomposition is implemented by the Gelfand transform; for $v \in L^{2}\left(\mathbb{R}^{d-1}\right)$ set

$$
(\mathcal{U} v)_{\theta}(\widetilde{x})=\sum_{l \in \Gamma} \mathrm{e}^{-\mathrm{i} \theta \cdot(\widetilde{x}+l)} v(\widetilde{x}+l), \quad \theta \in \mathcal{O}^{\dagger}, \widetilde{x} \in \mathbb{R}^{d-1} .
$$

This expression is clearly $\Gamma$-periodic in $\widetilde{x}$ so can be viewed as a function on $\mathbb{T}$; in fact $\mathcal{U}$ is a unitary mapping $L^{2}\left(\mathbb{R}^{d-1}\right) \rightarrow L^{2}\left(\mathcal{O}^{\dagger}, L^{2}(\mathbb{T})\right)$.

Let $u \in L^{2}\left(\mathbb{R}_{+}^{d}\right)$. For each $\theta \in \mathcal{O}^{\dagger}$ and $t>0$ set $\phi_{\theta}(\cdot, t)=(\mathcal{U} u(\cdot, t))_{\theta}$, considered as an element of $L^{2}(\mathbb{T})$.

Lemma 2.1. Suppose $u$ is a distributional solution of (1.1) on $\mathbb{R}_{+}^{d}$ and satisfies (1.2) for some $\kappa \geq 0$ and $\lambda>0$. Then $\phi_{\theta} \in \mathcal{X}_{\mathrm{loc}}^{2}$ with $\left(\nabla_{t}^{2}-H_{\theta}\right) \phi_{\theta}=V_{t} \phi_{\theta}$ and

$$
\int_{1}^{\infty} \mathrm{e}^{2 \lambda t}\left\|\phi_{\theta}(\cdot, t)\right\|_{L^{2}(\mathbb{T})}^{2} \mathrm{~d} t<+\infty
$$


for almost all $\theta \in \mathcal{O}^{\dagger}$. If $\kappa>(d-1) / 2$ then the same conclusion holds for all $\theta \in \mathcal{O}^{\dagger}$, while $\phi_{\theta}$ depends continuously on $\theta$.

Proof. Choose a basis $\left\{\mathbf{e}_{1}, \ldots, \mathbf{e}_{d-1}\right\}$ for $\Gamma$ corresponding to the unit cell $\mathcal{O}$. Set

$$
\mathcal{P}=\left\{r_{1} \mathbf{e}_{1}+\cdots+r_{d-1} \mathbf{e}_{d-1}: r_{1}, \ldots, r_{d-1} \in(-1 / 2,3 / 2)\right\}
$$

so $\bigcup_{l \in \Gamma}(\mathcal{P}+l)$ is a $2^{d-1}$-fold covering of $\mathbb{R}^{d-1}$. For any $l \in \Gamma$ and $s \geq 1$ let $\mathcal{O}_{l, s}=(\mathcal{O}+l) \times(s, s+1)$ and $\mathcal{P}_{l, s}=(\mathcal{P}+l) \times(s-1, s+2)$. Note that $\mathcal{O}_{l, s} \subset \subset \mathcal{P}_{l, s}$.

From (1.2) we get $u \in L^{2}\left(\mathbb{R}_{+}^{d}\right)$ (recall that $\kappa \geq 0$ and $\lambda>0$ ), while $\Delta u=(V-E) u$ as distributions and $V$ is uniformly bounded. It follows that $u \in H_{\text {loc }}^{2}\left(\mathbb{R}_{+}^{d}\right)$ and

$$
\|u\|_{H^{2}\left(\mathcal{O}_{l, s}\right)} \leq C_{1}\|u\|_{L^{2}\left(\mathcal{P}_{l, s}\right)}
$$

for some constant $C_{1}$ which is independent of $l \in \Gamma$ and $s \geq 1$. This uniformity leads to $u \in H^{2}\left(\mathbb{R}^{d-1} \times(1, \infty)\right)$. Applying the Gelfand transform we get

$$
(t, \theta) \mapsto(\mathcal{U} u(\cdot, t))_{\theta}=\phi_{\theta}(t, \cdot) \in \bigcap_{j=0,1,2} H^{j}\left((1, \infty) \times \mathcal{O}^{\dagger}, H^{2-j}(\mathbb{T})\right)
$$

while, using the definition of $H_{\theta}$ and the $\Gamma$-periodicity of $V$,

$$
\begin{aligned}
\left(\nabla_{t}^{2}-H_{\theta}\right) \phi_{\theta}(\widetilde{x}, t) & =\sum_{l \in \Gamma} \mathrm{e}^{-\mathrm{i} \theta \cdot(\widetilde{x}+l)}\left(\nabla_{t}^{2}-\left(-i \nabla_{\widetilde{x}}+\theta-\theta\right)^{2}+E\right) u(\widetilde{x}+l, t) \\
& =\sum_{l \in \Gamma} \mathrm{e}^{-\mathrm{i} \theta \cdot(\widetilde{x}+l)}(\Delta+E) u(\widetilde{x}+l, t) \\
& =V(\widetilde{x}, t) \sum_{l \in \Gamma} \mathrm{e}^{-\mathrm{i} \theta \cdot(\widetilde{x}+l)} u(\widetilde{x}+l, t)=V_{t}(\widetilde{x}) \phi_{\theta}(\widetilde{x}, t)
\end{aligned}
$$

as elements of $L^{2}\left((1, \infty) \times \mathcal{O}^{\dagger}, L^{2}(\mathbb{T})\right)$. Fubini's theorem then implies

$$
\phi_{\theta} \in \bigcap_{j=0,1,2} H^{j}\left((1, \infty), H^{2-j}(\mathbb{T})\right) \subset \mathcal{X}_{\mathrm{loc}}^{2},
$$

with $\left(\nabla_{t}^{2}-H_{\theta}\right) \phi_{\theta}=V_{t} \phi_{\theta}$ as elements of $\mathcal{X}_{\mathrm{loc}}^{0}$, for almost all $\theta \in \mathcal{O}^{\dagger}$.

Since $\mathcal{U}$ is unitary and $\kappa \geq 0$ (1.2) gives

$$
\int_{\mathcal{O}^{\dagger}} \int_{0}^{\infty} \int_{\mathbb{T}} \mathrm{e}^{2 \lambda t}\left|\phi_{\theta}(\widetilde{x}, t)\right|^{2} \mathrm{~d} \widetilde{x} \mathrm{~d} t \mathrm{~d} \theta=\int_{\mathbb{R}_{+}^{d}} \mathrm{e}^{2 \lambda t}|u(\widetilde{x}, t)|^{2} \mathrm{~d} \widetilde{x} \mathrm{~d} t<+\infty .
$$

This leads to (2.1) for almost all $\theta \in \mathcal{O}^{\dagger}$.

Now suppose (1.2) holds for some $\kappa>(d-1) / 2$. Then $C_{2}=\sum_{l \in \Gamma}\langle l\rangle^{-2 \kappa}<+\infty$. With $w=\sup \{|\widetilde{x}|: \widetilde{x} \in \mathcal{O}\}$ we also have

$$
\widetilde{x} \in \mathcal{P}+l \Longrightarrow|l| \leq|\widetilde{x}|+\frac{3}{2} w \Longrightarrow\langle l\rangle^{2 \kappa} \leq C_{3}\langle\widetilde{x}\rangle^{2 \kappa}
$$

where $C_{3}=\left(\max \left\{1+9 w^{2} / 2,2\right\}\right)^{\kappa}$. Let $\theta \in \mathcal{O}^{\dagger}$. For any $s \geq 1(2.2)$ then gives

$$
\begin{aligned}
\left\|\phi_{\theta}\right\|_{H^{2}(\mathbb{T} \times(s, s+1))} & \leq \sum_{l \in \Gamma}\left\|\mathrm{e}^{-\mathrm{i} \theta \cdot \widetilde{x}}\right\|_{C^{2}(\mathcal{O}+l)}\|u\|_{H^{2}\left(\mathcal{O}_{l, s}\right)} \\
& \leq C_{1} C_{4} \sum_{l \in \Gamma}\|u\|_{L^{2}\left(\mathcal{P}_{l, s}\right)} \leq C_{1} C_{4}\left(C_{2} N_{s}\right)^{1 / 2}
\end{aligned}
$$


where $C_{4}=\sup _{\theta^{\prime} \in \mathcal{O}^{\dagger}}\left\|\mathrm{e}^{-\mathrm{i} \theta^{\prime} \cdot \widetilde{x}}\right\|_{C^{2}\left(\mathbb{R}^{d-1}\right)}$ and

$$
\begin{aligned}
N_{s}=\sum_{l \in \Gamma}\langle l\rangle^{2 \kappa}\|u\|_{L^{2}\left(\mathcal{P}_{l, s}\right)}^{2} & \leq C_{3} \sum_{l \in \Gamma} \int_{s-1}^{s+2} \int_{\mathcal{P}+l}\langle\widetilde{x}\rangle^{2 \kappa}|u(\widetilde{x}, t)|^{2} \mathrm{~d} \widetilde{x} \mathrm{~d} t \\
& =2^{d-1} C_{3} \int_{s-1}^{s+2} \int_{\mathbb{R}^{d-1}}\langle\widetilde{x}\rangle^{2 \kappa}|u(\widetilde{x}, t)|^{2} \mathrm{~d} \widetilde{x} \mathrm{~d} t .
\end{aligned}
$$

Now $N_{s}<+\infty$ by (1.2), so $\phi_{\theta} \in \mathcal{X}_{\mathrm{loc}}^{2}$. A simpler version of this argument also gives

$$
\begin{aligned}
\int_{1}^{\infty} \int_{\mathbb{T}} \mathrm{e}^{2 \lambda t}\left|\phi_{\theta}(\widetilde{x}, t)\right|^{2} \mathrm{~d} \widetilde{x} \mathrm{~d} t & \leq C_{2} \sum_{s \in \mathbb{N}} \mathrm{e}^{2 \lambda(s+1)} \sum_{l \in \Gamma}\langle l\rangle^{2 \kappa}\|u\|_{L^{2}\left(\mathcal{O}_{l, s}\right)}^{2} \\
& \leq C_{2} C_{3} \mathrm{e}^{2 \lambda} \int_{1}^{\infty} \int_{\mathbb{R}^{d-1}}\langle\widetilde{x}\rangle^{2 \kappa} \mathrm{e}^{2 \lambda t}|u(\widetilde{x}, t)|^{2} \mathrm{~d} \widetilde{x} \mathrm{~d} t<+\infty
\end{aligned}
$$

For $l \in \Gamma$ and $\theta, \theta^{\prime} \in \mathcal{O}^{\dagger}$ set $\delta_{l}\left(\theta, \theta^{\prime}\right)=\left\|\mathrm{e}^{-\mathrm{i} \theta \cdot(\widetilde{x}+l)}-\mathrm{e}^{-\mathrm{i} \theta^{\prime} \cdot(\widetilde{x}+l)}\right\|_{C^{2}(\mathcal{O})}$. Arguing as above,

$$
\left\|\phi_{\theta}-\phi_{\theta^{\prime}}\right\|_{H^{2}(\mathbb{T} \times(s, s+1))}^{2} \leq C_{1}^{2} C_{2} \sum_{l \in \Gamma} \delta_{l}\left(\theta, \theta^{\prime}\right)^{2}\langle l\rangle^{2 \kappa}\|u\|_{L^{2}\left(\mathcal{P}_{l, s}\right)}^{2} .
$$

For fixed $l \in \Gamma, \delta_{l}\left(\theta, \theta^{\prime}\right) \leq 2 C_{4}$ and $\delta_{l}\left(\theta, \theta^{\prime}\right) \rightarrow 0$ as $\left|\theta-\theta^{\prime}\right| \rightarrow 0$. Since $N_{s}<+\infty$ dominated convergence then gives $\left\|\phi_{\theta}-\phi_{\theta^{\prime}}\right\|_{H^{2}(\mathbb{T} \times(s, s+1))} \rightarrow 0$ as $\left|\theta-\theta^{\prime}\right| \rightarrow 0$.

It is straightforward to check that

$$
\operatorname{spec}\left(H_{\theta}\right)=\left\{|k+\theta|^{2}-E: k \in \Gamma^{\dagger}\right\} .
$$

The next result establishes a key part of the hypothesis in theorem 1.4.

Proposition 2.2. Let $\theta \in \mathcal{O}^{\dagger}$. If $d \geq 3$ suppose that $\Gamma$ is a rational lattice (in $\mathbb{R}^{d-1}$ ) while $\theta$ has rational coordinates (with respect to a basis of $\Gamma^{\dagger}$ ).

(i) If $d=2$ or 3 then $\operatorname{spec}\left(H_{\theta}\right)$ contains arbitrarily large positive gaps.

(ii) If $d \geq 4$ then there exists $\delta>0$ such that $\operatorname{spec}\left(H_{\theta}\right)$ contains infinitely many positive gaps of length at least $\delta$.

When $d=3$ we need non-trivial information about the gaps in the values realised by a binary quadratic form. This is taken from [10] and was previously observed in [9] for the special case $\Gamma=(2 \pi \mathbb{Z})^{2}$.

Proof. If $d=2$ we have $\Gamma^{\dagger}=f \mathbb{Z}$ and $\theta \in[0, f)$ for some $f \in \mathbb{R}_{+}$. Then $\operatorname{spec}\left(H_{\theta}\right)=$ $\left\{(m f+\theta)^{2}-E: m \in \mathbb{Z}\right\}$; the existence of arbitrarily large gaps follows easily.

Now suppose $d \geq 3$. Choose a basis $\left\{\mathbf{f}_{1}, \ldots, \mathbf{f}_{d-1}\right\}$ for the lattice $\Gamma^{\dagger} \subset \mathbb{R}^{d-1}$ corresponding to the unit cell $\mathcal{O}^{\dagger}$. If $k \in \Gamma^{\dagger}$ and $\theta \in \mathcal{O}^{\dagger}$ we can write

$$
k=m_{1} \mathbf{f}_{1}+\cdots+m_{d-1} \mathbf{f}_{d-1} \quad \text { and } \quad \theta=\mu_{1} \mathbf{f}_{1}+\cdots+\mu_{d-1} \mathbf{f}_{d-1}
$$

for some $m_{i} \in \mathbb{Z}$ and $\mu_{i} \in[0,1), i=1, \ldots, d-1$. Since $\Gamma$ and hence $\Gamma^{\dagger}$ are rational, we can find $\sigma>0$ and a positive definite integral quadratic form $\mathbf{q}$ so that

$$
|k+\theta|^{2}=\sigma \mathbf{q}\left(m_{1}+\mu_{1}, \ldots, m_{d-1}+\mu_{d-1}\right)
$$


when $k \in \Gamma^{\dagger}$ and $\theta \in \mathcal{O}^{\dagger}$ are as given in (2.3). Let $Q=\left\{\mathbf{q}(\mathbf{m}): \mathbf{m} \in \mathbb{Z}^{d-1}\right\} \subseteq \mathbb{Z}$ denote the values of $\mathbf{q}$ realised by integer arguments.

Now suppose $\theta=\mu_{1} \mathbf{f}_{1}+\cdots+\mu_{d-1} \mathbf{f}_{d-1} \in \mathcal{O}^{\dagger}$ has rational coefficients. Thus we can write $\mu_{i}=r_{i} / l$ for some $l \in \mathbb{N}$ and $r_{i} \in\{0, \ldots, l-1\}, i=1, \ldots, d-1$. Then

$$
|k+\theta|^{2}=\frac{\sigma}{l^{2}} \mathbf{q}\left(l m_{1}+r_{1}, \ldots, l m_{d-1}+r_{d-1}\right) \in \frac{\sigma}{l^{2}} Q .
$$

Hence $\operatorname{spec}\left(H_{\theta}\right)+E \subseteq \sigma Q / l^{2} \subseteq \sigma \mathbb{Z} / l^{2}$; part (ii) follows.

If $d=3$ then $\mathbf{q}$ is a positive definite binary quadratic form. By [10, theorem 2] there exists $C_{5}>0$ such that

$$
\#\{n \in Q: n \leq N\} \sim \frac{C_{5} N}{\sqrt{\log N}} \text { as } N \rightarrow+\infty .
$$

The existence of arbitrarily large positive gaps in $Q$, and thus $\operatorname{spec}\left(H_{\theta}\right)$, follows.

Theorem 1.1 is now a straightforward corollary of theorem 1.4.

Proof of theorem 1.1. Suppose $d \geq 3$ and (1.2) holds for some $\kappa>(d-1) / 2$ and all $\lambda>0$. If $\theta \in \mathcal{O}^{\dagger}$ lemma 2.1 shows $\phi_{\theta} \in \mathcal{X}_{\text {loc }}^{2}$ satisfies $\left(\nabla_{t}^{2}-H_{\theta}\right) \phi_{\theta}=V_{t} \phi_{\theta}$ and

$$
\int_{1}^{\infty} \mathrm{e}^{2 \lambda t}\left\|\phi_{\theta}(\cdot, t)\right\|_{L^{2}(\mathbb{T})}^{2} \mathrm{~d} t<+\infty
$$

for all $\lambda>0$. If $\Gamma^{\dagger}$ is rational and $\theta \in \mathcal{O}^{\dagger}$ has rational coordinates with respect to $\Gamma^{\dagger}$, proposition 2.2 and (a translated version of) theorem 1.4 then give $\phi_{\theta}(\cdot, t) \equiv 0$ for all $t>1$. However the set of $\theta \in \mathcal{O}^{\dagger}$ with rational coordinates is dense, while $\phi_{\theta}$ depends continuously on $\theta$ by lemma 2.1 . It follows that $\phi_{\theta} \equiv 0$ on $\mathbb{T} \times(1, \infty)$ for all $\theta \in \mathcal{O}^{\dagger}$.

Now let $\widetilde{x} \in \mathbb{R}^{d-1}$ and choose $\widetilde{x}^{\prime} \in \mathcal{O}$ and $l^{\prime} \in \Gamma$ with $\widetilde{x}=\widetilde{x}^{\prime}+l^{\prime}$. Then, for $t>1$,

$$
\begin{aligned}
0 & =\frac{1}{\left|\mathcal{O}^{\dagger}\right|} \int_{\mathcal{O}^{\dagger}} \mathrm{e}^{\mathrm{i} \theta \cdot \widetilde{x}} \phi_{\theta}\left(\widetilde{x}^{\prime}, t\right) \mathrm{d} \theta \\
& =\frac{1}{\left|\mathcal{O}^{\dagger}\right|} \int_{\mathcal{O}^{\dagger}} \sum_{l \in \Gamma} \mathrm{e}^{\mathrm{i} \theta \cdot\left(l^{\prime}-l\right)} u\left(\widetilde{x}^{\prime}+l, t\right) \mathrm{d} \theta=\sum_{l \in \Gamma} \delta_{l l^{\prime}} u\left(\widetilde{x}^{\prime}+l, t\right)=u(\widetilde{x}, t) .
\end{aligned}
$$

Thus $u \equiv 0$ on $\mathbb{R}^{d-1} \times(1, \infty) \subset \mathbb{R}_{+}^{d}$. Unique continuation (see [11, theorem XIII.63], for example) then shows $u$ is trivial on $\mathbb{R}_{+}^{d}$.

The case $d=2$ can be handled similarly; in this case we get $\phi_{\theta} \equiv 0$ on $\mathbb{T} \times(1, \infty)$ for almost all $\theta \in \mathcal{O}^{\dagger}$; this is sufficient to allow the reconstruction of $u$ as in (2.4).

\section{General result}

The Carleman type estimates we use for theorems 1.3 and 1.4 are stated in propositions 3.1 and 3.2 respectively; their proofs are deferred to $\S 4$. For convenience choose $\alpha \geq 0$ with $A+\alpha I \geq 0$; in particular $\operatorname{spec}(A) \subseteq[-\alpha,+\infty)$.

Proposition 3.1. Let $\phi \in C_{0}^{2}\left(\mathbb{R}_{+}, X_{2}\right)$ and choose $\varepsilon>0$ so that $\phi(t)=0$ for $t<\varepsilon$. If $\lambda \geq \varepsilon^{-4 / 3}$ then

$$
\lambda^{3} \int_{0}^{\infty} \mathrm{e}^{2 \lambda t^{4 / 3}}\|\phi(t)\|_{X}^{2} \mathrm{~d} t \leq \int_{0}^{\infty} \mathrm{e}^{2 \lambda t^{4 / 3}}\left\|\left(\nabla_{t}^{2}-A\right) \phi(t)\right\|_{X}^{2} \mathrm{~d} t .
$$


Proposition 3.2. Suppose $\left(\mu^{2}, \nu^{2}\right) \cap \operatorname{spec}(A)=\emptyset$ for some $0<\mu<\nu$ with $3 \mu^{2}>\alpha$. Set $\lambda=(\mu+\nu) / 2$ and $\gamma=\nu-\mu$. For any $\phi \in C_{0}^{2}\left(\mathbb{R}_{+}, X_{2}\right)$ we have

$$
\frac{\mu^{2} \gamma^{2}}{4} \int_{0}^{\infty} \mathrm{e}^{2 \lambda t}\|\phi(t)\|_{X}^{2} \mathrm{~d} t \leq \int_{0}^{\infty} \mathrm{e}^{2 \lambda t}\left\|\left(\nabla_{t}^{2}-A\right) \phi(t)\right\|_{X}^{2} \mathrm{~d} t .
$$

To apply these Carleman estimates we need to use the bounds on $\|\phi(t)\|_{X}$ given by (1.3) or (1.4) to obtain similar bounds for $\left\|\nabla_{t} \phi(t)\right\|_{X}$; this can be done using the 'elliptic regularity' of the operator $\nabla_{t}^{2}-A$.

Lemma 3.3. Let $\phi \in \mathcal{X}_{\mathrm{loc}}^{2}$ and suppose $\left\|\left(\nabla_{t}^{2}-A\right) \phi(t)\right\|_{X} \leq \beta\|\phi(t)\|_{X}$ for some $\beta>0$ and all $t>0$. Let $\sigma \geq 1$. If

$$
\int_{0}^{\infty} \mathrm{e}^{2 \lambda t^{\sigma}}\|\phi(t)\|_{X}^{2} \mathrm{~d} t<+\infty
$$

for all $\lambda>0$ then

$$
\int_{\varepsilon}^{\infty} \mathrm{e}^{2 \lambda t^{\sigma}}\left\|\nabla_{t} \phi(t)\right\|_{X}^{2} \mathrm{~d} t<+\infty
$$

for all $\varepsilon, \lambda>0$.

Proof. Set $N=(A+\alpha I)^{1 / 2}$. For any $h \in C_{0}^{\infty}\left(\mathbb{R}_{+}\right)$we have

$$
\begin{aligned}
\int_{0}^{\infty} & h^{2}\left[\left\|\nabla_{t} \phi\right\|_{X}^{2}+\|N \phi\|_{X}^{2}\right]=-\int_{0}^{\infty} \operatorname{Re}\left[h^{2}\left\langle\phi,\left(\nabla_{t}^{2}-N^{2}\right) \phi\right\rangle_{X}+2 h h^{\prime}\left\langle\phi, \nabla_{t} \phi\right\rangle_{X}\right] \\
& \leq \int_{0}^{\infty} h^{2}\left|\left\langle\phi,\left(\alpha I-\left(\nabla_{t}^{2}-A\right)\right) \phi\right\rangle_{X}\right|+2 \int_{0}^{\infty}\left|h h^{\prime}\left\langle\phi, \nabla_{t} \phi\right\rangle_{X}\right| \\
& \leq(\alpha+\beta) \int_{0}^{\infty} h^{2}\|\phi\|_{X}^{2}+2 \int_{0}^{\infty}\left(h^{\prime}\right)^{2}\|\phi\|_{X}^{2}+\frac{1}{2} \int_{0}^{\infty} h^{2}\left\|\nabla_{t} \phi\right\|_{X}^{2}
\end{aligned}
$$

since $4\left|h h^{\prime}\left\langle\phi, \nabla_{t} \phi\right\rangle_{X}\right| \leq 4\left(h^{\prime}\right)^{2}\|\phi\|_{X}^{2}+h^{2}\left\|\nabla_{t} \phi\right\|_{X}^{2}$. If $\varepsilon>0$ we can then choose $h$ to be an appropriate translate of a function with support in $(-\varepsilon, 1+\varepsilon)$ and value 1 on $(0,1)$ to find $C_{6}>0$ such that

$$
\int_{s}^{s+1}\left\|\nabla_{t} \phi\right\|_{X}^{2} \leq C_{6} \int_{s-\varepsilon}^{s+1+\varepsilon}\|\phi\|_{X}^{2}
$$

for all $s \geq \varepsilon$. If $\lambda^{\prime}>\lambda>0$ we can find $C_{7}>0$ with $\lambda(n+1+\varepsilon)^{\sigma}-\lambda^{\prime} n^{\sigma} \leq C_{7}$ for all $n \geq 0$. Then

$$
\begin{aligned}
\int_{\varepsilon}^{\infty} \mathrm{e}^{2 \lambda t^{\sigma}}\left\|\nabla_{t} \phi\right\|_{X}^{2} & \leq \sum_{n=0}^{\infty} \mathrm{e}^{2 \lambda(n+1+\varepsilon)^{\sigma}} \int_{n+\varepsilon}^{n+1+\varepsilon}\left\|\nabla_{t} \phi\right\|_{X}^{2} \\
& \leq C_{6} e^{2 C_{7}} \sum_{n=0}^{\infty} \mathrm{e}^{2 \lambda^{\prime} n^{\sigma}} \int_{n}^{n+1+2 \varepsilon}\|\phi\|_{X}^{2} \\
& \leq C_{6} e^{2 C_{7}} \sum_{n=0}^{\infty} \int_{n}^{n+1+2 \varepsilon} \mathrm{e}^{2 \lambda^{\prime} t^{\sigma}}\|\phi\|_{X}^{2} \\
& \leq 2(1+\varepsilon) C_{6} e^{2 C_{7}} \int_{0}^{\infty} \mathrm{e}^{2 \lambda^{\prime} t^{\sigma}}\|\phi\|_{X}^{2} .
\end{aligned}
$$

The result follows. 
Proof of theorem 1.3. Set $\psi=\left(\nabla_{t}^{2}-A\right) \phi \in \mathcal{X}_{\mathrm{loc}}^{0}$. Let $\varepsilon \in(0,1)$ and suppose $\lambda>$ $\left(2 \beta^{2}\right)^{1 / 3},(\varepsilon / 2)^{-4 / 3}$. For each $R>1$ choose a cut-off function $h_{R} \in C_{0}^{\infty}\left(\mathbb{R}_{+}\right)$with $\operatorname{supp}\left(h_{R}\right) \subseteq(\varepsilon / 2,2 R), h_{R}(t)=1$ for $t \in[\varepsilon, R], h_{R}(t)$ independent of $R$ for $t \leq \varepsilon$,

$$
\sup _{t \in[R, 2 R]} h_{R}^{\prime}(t) \leq \frac{2}{R} \quad \text { and } \quad \sup _{t \in[R, 2 R]} h_{R}^{\prime \prime}(t) \leq \frac{8}{R^{2}}
$$

By using a mollifier (for example) we can find an approximating sequence for $h_{R} \phi$ in $C_{0}^{2}\left(\mathbb{R}^{+}, X_{2}\right)$; we may further assume elements of this sequence are supported in $[\varepsilon / 2, \infty)$. Apply proposition 3.1 to elements of this sequence; taking the limit and noting that $\left(\nabla_{t}^{2}-A\right)\left(h_{R} \phi\right)=h_{R}^{\prime \prime} \phi+2 h_{R}^{\prime} \nabla_{t} \phi+h_{R} \psi$, we get the estimate

$$
\begin{aligned}
& \lambda^{3} \int_{0}^{\infty} \mathrm{e}^{2 \lambda t^{4 / 3}} h_{R}^{2}\|\phi\|_{X}^{2} \leq \int_{0}^{\infty} \mathrm{e}^{2 \lambda t^{4 / 3}}\left\|h_{R}^{\prime \prime} \phi+2 h_{R}^{\prime} \nabla_{t} \phi+h_{R} \psi\right\|_{X}^{2} \\
& \quad \leq 2 \beta^{2} \int_{0}^{\infty} \mathrm{e}^{2 \lambda t^{4 / 3}} h_{R}^{2}\|\phi\|_{X}^{2}+\int_{0}^{\infty} \mathrm{e}^{2 \lambda t^{4 / 3}}\left[4\left(h_{R}^{\prime \prime}\right)^{2}\|\phi\|_{X}^{2}+16\left(h_{R}^{\prime}\right)^{2}\left\|\nabla_{t} \phi\right\|_{X}^{2}\right] .
\end{aligned}
$$

Rearranging and using (3.3) we get

$$
\left(\lambda^{3}-2 \beta^{2}\right) \int_{\varepsilon}^{R} \mathrm{e}^{2 \lambda t^{4 / 3}}\|\phi\|_{X}^{2} \leq C_{8} \mathrm{e}^{2 \lambda \varepsilon^{4 / 3}}+\rho(R)
$$

where

$$
C_{8}=\int_{0}^{\varepsilon}\left[4\left(h_{R}^{\prime \prime}\right)^{2}\|\phi\|_{X}^{2}+16\left(h_{R}^{\prime}\right)^{2}\left\|\nabla_{t} \phi\right\|_{X}^{2}\right],
$$

which is independent of $R$ and $\lambda$, and

$$
\rho(R)=\frac{2^{8}}{R^{4}} \int_{R}^{2 R} \mathrm{e}^{2 \lambda t^{4 / 3}}\|\phi\|_{X}^{2}+\frac{2^{6}}{R^{2}} \int_{R}^{2 R} \mathrm{e}^{2 \lambda t^{4 / 3}}\left\|\nabla_{t} \phi\right\|_{X}^{2} .
$$

However, our hypothesis and lemma 3.3(i) give

$$
\int_{0}^{\infty} \mathrm{e}^{2 \lambda t^{4 / 3}}\|\phi\|_{X}^{2}, \int_{\varepsilon / 2}^{\infty} \mathrm{e}^{2 \lambda t^{4 / 3}}\left\|\nabla_{t} \phi\right\|_{X}^{2}<+\infty .
$$

Thus $\rho(R) \rightarrow 0$ as $R \rightarrow \infty$. It follows that

$$
\left(\lambda^{3}-2 \beta^{2}\right) \int_{\varepsilon}^{\infty} \mathrm{e}^{2 \lambda t^{4 / 3}}\|\phi\|_{X}^{2} \leq C_{8} \mathrm{e}^{2 \lambda \varepsilon^{4 / 3}} .
$$

For any $\varepsilon_{1}>\varepsilon$ we then get

$$
\left(\lambda^{3}-2 \beta^{2}\right) \int_{\varepsilon_{1}}^{\infty}\|\phi\|_{X}^{2} \leq C_{8} \mathrm{e}^{-2 \lambda\left(\varepsilon_{1}^{4 / 3}-\varepsilon^{4 / 3}\right)} .
$$

However this inequality will be contradicted for sufficiently large $\lambda$ if $\int_{\varepsilon_{1}}^{\infty}\|\phi\|_{X}^{2}>0$. Hence $\phi(t)=0$ for $t>\varepsilon$. Since $\varepsilon \in(0,1)$ was arbitrary the result follows. 
Proof of theorem 1.4. Define a non-increasing function by $\beta(t)=\sup _{t^{\prime}>t} b\left(t^{\prime}\right)$ for $t \geq 0$. Suppose $\left(\mu^{2}, \nu^{2}\right) \cap \operatorname{spec}(A)=\emptyset$ for some $0<\mu<\nu$ with $3 \mu^{2}>\alpha$. Set $\lambda=(\mu+\nu) / 2$ and $\gamma=\nu-\mu$. Suppose $\mu^{2} \gamma^{2}>8 \beta^{2}\left(t_{0}\right)$ for some $t_{0} \geq 0$. We can now emulate the proof of theorem 1.3; following the argument as far as (3.4) we get

$$
\left(\frac{\mu^{2} \gamma^{2}}{4}-2 \beta^{2}\left(t_{0}\right)\right) \int_{t_{2}}^{\infty}\|\phi\|_{X}^{2} \leq C_{9} \mathrm{e}^{-2 \lambda\left(t_{2}-t_{1}\right)}
$$

for any $t_{2}>t_{1}>t_{0}$, where $C_{9}$ is a constant which is independent of $\mu, \nu, \gamma$ and $\lambda$.

Our hypothesis gives a sequence of disjoint intervals $\left(\mu_{n}^{2}, \nu_{n}^{2}\right), n=1,2, \ldots$, in $\mathbb{R}_{+} \backslash \operatorname{spec}(A)$ with either (i) $\nu_{n}^{2}-\mu_{n}^{2} \rightarrow \infty$ as $n \rightarrow \infty$, or (ii) $\nu_{n}^{2}-\mu_{n}^{2} \geq \delta$ for all $n$ and $\beta(t) \rightarrow 0$ as $t \rightarrow \infty$. We may further assume $\mu_{n}$ is increasing and $\nu_{n} \leq 3 \mu_{n}$ for all $n$. Then $\lambda_{n}=\left(\nu_{n}+\mu_{n}\right) / 2 \rightarrow \infty$ as $n \rightarrow \infty$, while

$$
\nu_{n}^{2}-\mu_{n}^{2}=\left(\nu_{n}+\mu_{n}\right)\left(\nu_{n}-\mu_{n}\right) \leq 4 \mu_{n}\left(\nu_{n}-\mu_{n}\right)=4 \mu_{n} \gamma_{n}
$$

for all $n$. We complete the argument for the two cases separately.

(i) In this case $\mu_{n} \gamma_{n} \rightarrow \infty$ as $n \rightarrow \infty$. Taking $t_{0}=0$ we will contradict (3.5) for sufficiently large $n$ unless $\int_{t_{2}}^{\infty}\|\phi\|_{X}^{2}=0$ for all $t_{2}>0$. Hence $\phi \equiv 0$ on $\mathbb{R}_{+}$.

(ii) Choose $t_{0}$ so $\beta^{2}\left(t_{0}\right)<2^{-7} \delta^{2}$. Then $\mu_{n}^{2} \gamma_{n}^{2} / 4 \geq 2^{-6} \delta^{2}>2 \beta^{2}\left(t_{0}\right)$ for all $n$. Since $\lambda_{n} \rightarrow \infty(3.5)$ will be contradicted for sufficiently large $n$ unless $\int_{t_{2}}^{\infty}\|\phi\|_{X}^{2}=0$ for all $t_{2}>t_{0}$. Thus $\phi(t)=0$ for $t>t_{0}$. However (1.4) then implies (1.3), so $\phi \equiv 0$ on $\mathbb{R}_{+}$by theorem 1.3

\section{Carleman estimates}

Proof of proposition 3.1. Define $\Omega, \omega: \mathbb{R}_{+} \rightarrow \mathbb{R}$ by $\Omega(t)=\lambda t^{4 / 3}$ and $\omega=\Omega^{\prime}$. Then

$$
\mathrm{e}^{\Omega}\left(\nabla_{t}^{2}-A\right)\left(\mathrm{e}^{-\Omega} \cdot\right)=\nabla_{t}^{2}-A_{\omega}-L
$$

where $A_{\omega}=A-\omega^{2}$ and $L=2 \omega \nabla_{t}+\omega^{\prime}$. If $\psi \in C_{0}^{2}\left(\mathbb{R}_{+}, X_{2}\right)$ then

$$
\begin{gathered}
\|L \psi\|_{X}^{2}=4 \omega^{2}\left\|\nabla_{t} \psi\right\|_{X}^{2}+\left(\omega^{2}\right)^{\prime} \nabla_{t}\|\psi\|_{X}^{2}+\left(\omega^{\prime}\right)^{2}\|\psi\|_{X}^{2}, \\
2 \operatorname{Re}\left\langle L \psi, \nabla_{t}^{2} \psi\right\rangle_{X}=2 \omega \nabla_{t}\left\|\nabla_{t} \psi\right\|_{X}^{2}+\omega^{\prime}\left(\nabla_{t}^{2}\|\psi\|_{X}^{2}-2\left\|\nabla_{t} \psi\right\|_{X}^{2}\right)
\end{gathered}
$$

and

$$
\begin{aligned}
2 \operatorname{Re}\left\langle L \psi, A_{\omega} \psi\right\rangle_{X} & =2 \omega \nabla_{t}\langle\psi, A \psi\rangle_{X}+2 \omega^{\prime}\langle\psi, A \psi\rangle_{X}-2 \omega^{3} \nabla_{t}\|\psi\|_{X}^{2}-2 \omega^{2} \omega^{\prime}\|\psi\|_{X}^{2} \\
& =2 \nabla_{t}\left(\omega\langle\psi, A \psi\rangle_{X}\right)-2 \nabla_{t}\left(\omega^{3}\|\psi\|_{X}^{2}\right)+\frac{4}{3}\left(\omega^{3}\right)^{\prime}\|\psi\|_{X}^{2} .
\end{aligned}
$$

Integration then leads to

$$
\begin{gathered}
\int_{0}^{\infty}\left\|\mathrm{e}^{\Omega}\left(\nabla_{t}^{2}-A\right)\left(\mathrm{e}^{-\Omega} \psi\right)\right\|_{X}^{2} \geq \int_{0}^{\infty}\left(\|L \psi\|_{X}^{2}-2 \operatorname{Re}\left\langle L \psi, \nabla_{t}^{2} \psi\right\rangle_{X}+2 \operatorname{Re}\left\langle L \psi, A_{\omega} \psi\right\rangle_{X}\right) \\
=\int_{0}^{\infty} 4\left(\omega^{2}+\omega^{\prime}\right)\left\|\nabla_{t} \psi\right\|_{X}^{2}+\int_{0}^{\infty}\left(\frac{4}{3}\left(\omega^{3}\right)^{\prime}-\left(\omega^{\prime}\right)^{2}-2 \omega \omega^{\prime \prime}-\omega^{\prime \prime \prime}\right)\|\psi\|_{X}^{2} .
\end{gathered}
$$

However $\omega^{\prime} \geq 0,\left(\omega^{3}\right)^{\prime}=(4 \lambda / 3)^{3} \geq 3 \lambda^{3} / 4$ and

$$
-\left(\left(\omega^{\prime}\right)^{2}+2 \omega \omega^{\prime \prime}+\omega^{\prime \prime \prime}\right)(t)=\frac{8}{81} \lambda t^{-4 / 3}\left(6 \lambda-5 t^{-4 / 3}\right),
$$

which is positive when $\lambda \geq t^{-4 / 3}$. Taking $\psi=\mathrm{e}^{\Omega} \phi$ now completes the result. 
Proof of proposition 3.2. Let $P_{-}$and $P_{+}$denote the orthogonal spectral projections of $A$ on $X$ corresponding to the intervals $\left[-\alpha, \mu^{2}\right]$ and $\left[\nu^{2},+\infty\right)$ respectively. Note that $P_{-}+P_{+}=I$. Set $\psi=\left(\nabla_{t}^{2}-A\right) \phi$. Denote the corresponding projections by $\phi_{ \pm}=P_{ \pm} \phi$ and $\psi_{ \pm}=P_{ \pm} \psi$; in particular, $\psi_{ \pm}=\left(\nabla_{t}^{2}-A\right) \phi_{ \pm}$. Introduce the operator $N=\left(A P_{+}\right)^{1 / 2}$; this commutes with $A, P_{ \pm}$and $\nabla_{t}$, and defines bounded maps $X_{2} \rightarrow X_{1}$ and $X_{1} \rightarrow X$.

To move from a second order equation to a system of first order ones we introduce spaces $\mathbf{X}_{j}=X_{j} \otimes \mathbb{C}^{2}$ for $j=0,1,2$, and put $\mathbf{X}=\mathbf{X}_{0}$. Setting

$$
\mathbf{Q}_{0}=\left(\begin{array}{cc}
P_{-} & 0 \\
0 & P_{-}
\end{array}\right), \quad \mathbf{Q}_{1}=\left(\begin{array}{cc}
P_{+} & 0 \\
0 & 0
\end{array}\right) \quad \text { and } \quad \mathbf{Q}_{2}=\left(\begin{array}{cc}
0 & 0 \\
0 & P_{+}
\end{array}\right)
$$

gives orthogonal projections on $\mathbf{X}$ with $\mathbf{Q}_{0}+\mathbf{Q}_{1}+\mathbf{Q}_{2}=\mathbf{I}$. Let

$$
\boldsymbol{\Phi}=\mathrm{e}^{\lambda t}\left(\begin{array}{c}
\nabla_{t} \phi+\left(\mu P_{-}+N\right) \phi \\
\nabla_{t} \phi-\left(\mu P_{-}+N\right) \phi
\end{array}\right) \quad \text { and } \quad \boldsymbol{\Psi}=\mathrm{e}^{\lambda t}\left(\begin{array}{c}
\psi \\
\psi
\end{array}\right) .
$$

For $j=0,1,2$ denote the corresponding projections by $\boldsymbol{\Phi}_{j}=\mathbf{Q}_{j} \boldsymbol{\Phi}$ and $\boldsymbol{\Psi}_{j}=\mathbf{Q}_{j} \mathbf{\Psi}$. Then

$$
\boldsymbol{\Phi}_{0}=\mathrm{e}^{\lambda t}\left(\begin{array}{c}
\left(\nabla_{t}+\mu\right) \phi_{-} \\
\left(\nabla_{t}-\mu\right) \phi_{-}
\end{array}\right), \quad \boldsymbol{\Phi}_{1}=\mathrm{e}^{\lambda t}\left(\begin{array}{c}
\left(\nabla_{t}+N\right) \phi_{+} \\
0
\end{array}\right)
$$

and

$$
\boldsymbol{\Phi}_{2}=\mathrm{e}^{\lambda t}\left(\begin{array}{c}
0 \\
\left(\nabla_{t}-N\right) \phi_{+}
\end{array}\right) .
$$

Now $\left(\nabla_{t} \pm \mu\right) \phi_{-} \in C_{0}^{1}\left(\mathbb{R}_{+}, X_{2}\right)$ and $\left(\nabla_{t} \pm N\right) \phi_{+} \in C_{0}^{1}\left(\mathbb{R}_{+}, X_{1}\right)$ so $\boldsymbol{\Phi}_{0} \in C_{0}^{1}\left(\mathbb{R}_{+}, \mathbf{X}_{2}\right)$ and $\boldsymbol{\Phi}_{1}, \boldsymbol{\Phi}_{2} \in C_{0}^{1}\left(\mathbb{R}_{+}, \mathbf{X}_{1}\right)$, while $\boldsymbol{\Psi}_{0}, \boldsymbol{\Psi}_{1}, \mathbf{\Psi}_{2} \in C_{0}^{0}\left(\mathbb{R}_{+}, \mathbf{X}\right)$. We also have

$\nabla_{t}\left(\nabla_{t} \pm \mu\right) \phi_{-}=\left(A \pm \mu \nabla_{t}\right) \phi_{-}+\psi_{-} \quad$ and $\quad \nabla_{t}\left(\nabla_{t} \pm N\right) \phi_{+}= \pm N\left(\nabla_{t} \pm N\right) \phi_{+}+\psi_{+}$.

It follows that $\nabla_{t} \boldsymbol{\Phi}_{j}=\mathbf{M}_{j} \boldsymbol{\Phi}_{j}+\boldsymbol{\Psi}_{j}$ for $j=0,1,2$, where

$$
\begin{gathered}
\mathbf{M}_{0}=\frac{1}{2 \mu}\left(\begin{array}{cc}
A+\mu^{2} & -A+\mu^{2} \\
A-\mu^{2} & -A-\mu^{2}
\end{array}\right) \mathbf{Q}_{0}+\lambda \mathbf{Q}_{0}, \\
\mathbf{M}_{1}=\left(\begin{array}{cc}
N & 0 \\
0 & 0
\end{array}\right)+\lambda \mathbf{Q}_{1} \text { and } \mathbf{M}_{2}=\left(\begin{array}{cc}
0 & 0 \\
0 & -N
\end{array}\right)+\lambda \mathbf{Q}_{2} .
\end{gathered}
$$

Therefore

$$
\begin{aligned}
\nabla_{t}\left\|\boldsymbol{\Phi}_{j}\right\|_{\mathbf{X}}^{2} & =\left\langle\nabla_{t} \boldsymbol{\Phi}_{j}, \boldsymbol{\Phi}_{j}\right\rangle_{\mathbf{X}}+\left\langle\boldsymbol{\Phi}_{j}, \nabla_{t} \boldsymbol{\Phi}_{j}\right\rangle_{\mathbf{X}} \\
& =\left\langle\boldsymbol{\Phi}_{j},\left(\mathbf{M}_{j}^{*}+\mathbf{M}_{j}\right) \boldsymbol{\Phi}_{j}\right\rangle_{\mathbf{X}}+2 \operatorname{Re}\left\langle\boldsymbol{\Psi}_{j}, \boldsymbol{\Phi}_{j}\right\rangle_{\mathbf{X}}
\end{aligned}
$$

Now $-3 \mu P_{-} \leq-\alpha \mu^{-1} P_{-} \leq \mu^{-1} A P_{-} \leq \mu P_{-}$and $N \geq \nu P_{+}$so

$$
\begin{aligned}
\mathbf{M}_{0}^{*}+\mathbf{M}_{0} & =\left(\begin{array}{cc}
\left(\mu^{-1} A+\mu+2 \lambda\right) P_{-} & 0 \\
0 & \left(-\mu^{-1} A-\mu+2 \lambda\right) P_{-}
\end{array}\right) \\
& \geq 2(\lambda-\mu)\left(\begin{array}{cc}
P_{-} & 0 \\
0 & P_{-}
\end{array}\right)=\gamma \mathbf{Q}_{0}
\end{aligned}
$$


while

$$
\mathbf{M}_{1}^{*}+\mathbf{M}_{1}=\left(\begin{array}{cc}
2 N+2 \lambda P_{+} & 0 \\
0 & 0
\end{array}\right) \geq 2(\nu+\lambda)\left(\begin{array}{cc}
P_{+} & 0 \\
0 & 0
\end{array}\right) \geq \gamma \mathbf{Q}_{1}
$$

For $j=0,1$ (4.1) then leads to

$$
\nabla_{t}\left\|\boldsymbol{\Phi}_{j}\right\|_{\mathbf{X}}^{2} \geq \gamma\left\|\boldsymbol{\Phi}_{j}\right\|_{\mathbf{X}}^{2}-2\left\|\boldsymbol{\Psi}_{j}\right\|_{\mathbf{X}}\left\|\boldsymbol{\Phi}_{j}\right\|_{\mathbf{X}} \geq \frac{\gamma}{2}\left\|\boldsymbol{\Phi}_{j}\right\|_{\mathbf{X}}^{2}-\frac{2}{\gamma}\left\|\boldsymbol{\Psi}_{j}\right\|_{\mathbf{X}}^{2} .
$$

Since $\left\|\boldsymbol{\Phi}_{j}\right\|_{\mathbf{X}}^{2} \in C_{0}^{1}\left(\mathbb{R}_{+}\right)$we can now integrate this inequality to get

$$
\frac{\gamma^{2}}{4} \int_{0}^{\infty}\left\|\boldsymbol{\Phi}_{j}\right\|_{\mathbf{X}}^{2} \leq \int_{0}^{\infty}\left\|\boldsymbol{\Psi}_{j}\right\|_{\mathbf{X}}^{2}
$$

for $j=0,1$. A simpler version of the above argument gives

$$
\mathbf{M}_{2}^{*}+\mathbf{M}_{2}=\left(\begin{array}{cc}
0 & 0 \\
0 & -2 N+2 \lambda P_{+}
\end{array}\right) \leq 2(\lambda-\nu)\left(\begin{array}{cc}
0 & 0 \\
0 & P_{+}
\end{array}\right)=-\gamma \mathbf{Q}_{2},
$$

so

$$
\nabla_{t}\left\|\boldsymbol{\Phi}_{2}\right\|_{\mathbf{X}}^{2} \leq-\gamma\left\|\boldsymbol{\Phi}_{2}\right\|_{\mathbf{X}}^{2}+2\left\|\mathbf{\Psi}_{2}\right\|_{\mathbf{X}}\left\|\boldsymbol{\Phi}_{2}\right\|_{\mathbf{X}} \leq-\frac{\gamma}{2}\left\|\boldsymbol{\Phi}_{2}\right\|_{\mathbf{X}}^{2}+\frac{2}{\gamma}\left\|\boldsymbol{\Psi}_{2}\right\|_{\mathbf{X}}^{2},
$$

and hence (4.2) for $j=2$. However

$$
\begin{aligned}
\left\|\boldsymbol{\Phi}_{0}\right\|_{\mathbf{X}}^{2}+ & \left\|\boldsymbol{\Phi}_{1}\right\|_{\mathbf{X}}^{2}+\left\|\boldsymbol{\Phi}_{2}\right\|_{\mathbf{X}}^{2} \\
= & \mathrm{e}^{2 \lambda t}\left(\left\|\left(\nabla_{t}+\mu\right) \phi_{-}\right\|_{X}^{2}+\left\|\left(\nabla_{t}-\mu\right) \phi_{-}\right\|_{X}^{2}\right. \\
& \left.\quad+\left\|\left(\nabla_{t}+N\right) \phi_{+}\right\|_{X}^{2}+\left\|\left(\nabla_{t}-N\right) \phi_{+}\right\|_{X}^{2}\right) \\
= & 2 \mathrm{e}^{2 \lambda t}\left(\left\|\nabla_{t} \phi_{-}\right\|_{X}^{2}+\mu^{2}\left\|\phi_{-}\right\|_{X}^{2}+\left\|\nabla_{t} \phi_{+}\right\|_{X}^{2}+\left\|N \phi_{+}\right\|_{X}^{2}\right) \\
\geq & 2 \mathrm{e}^{2 \lambda t}\left(\left\|\nabla_{t} \phi_{-}\right\|_{X}^{2}+\mu^{2}\left\|\phi_{-}\right\|_{X}^{2}+\left\|\nabla_{t} \phi_{+}\right\|_{X}^{2}+\nu^{2}\left\|\phi_{+}\right\|_{X}^{2}\right) \\
\geq & 2 \mathrm{e}^{2 \lambda t}\left(\left\|\nabla_{t} \phi\right\|_{X}^{2}+\mu^{2}\|\phi\|_{X}^{2}\right),
\end{aligned}
$$

while

$$
\left\|\boldsymbol{\Psi}_{0}\right\|_{\mathbf{X}}^{2}+\left\|\boldsymbol{\Psi}_{1}\right\|_{\mathbf{X}}^{2}+\left\|\mathbf{\Psi}_{2}\right\|_{\mathbf{X}}^{2}=2 \mathrm{e}^{2 \lambda t}\left(\left\|\psi_{-}\right\|_{X}^{2}+\left\|\psi_{+}\right\|_{X}^{2}\right)=2 \mathrm{e}^{2 \lambda t}\|\psi\|_{X}^{2} .
$$

These can be combined with (4.2) for $j=0,1,2$ to complete the result.

\section{Acknowledgements}

The author wishes to acknowledge the hospitality of the Isaac Newton Institute for Mathematical Sciences in Cambridge, UK, where this work was initiated during the programme Periodic and Ergodic Spectral Problems. The author also wishes to thank the referee for several useful comments and suggestions.

\section{References}

1 C. Bardos and M. Merigot. Asymptotic decay of the solution of a second-order elliptic equation in an unbounded domain. Applications to the spectral properties of a Hamiltonian. Proc. Roy. Soc. Edinburgh Sect. A76 (1977), 323-344. 
2 J. Cruz-Sampedro. Unique Continuation at Infinity of Solutions to Schrödinger Equations with Complex-Valued Potentials. Proc. Edinb. Math. Soc. 42 (1999), 143-153.

3 R. Froese, I. Herbst, M. Hoffmann-Ostenhof and T. Hoffmann-Ostenhof. $L^{2}$-Exponential Lower Bounds to Solutions of the Schrödinger Equation. Comm. Math. Phys. 87 (1982), $265-286$.

4 R. Froese, I. Herbst, M. Hoffmann-Ostenhof and T. Hoffmann-Ostenhof. On the absence of positive eigenvalues for one-body Schrödinger operators. J. d'Analyse 41 (1982) 272-284.

5 C. Kenig, L. Silvestre and J-N. Wang, On Landis' Conjecture in the Plane. Comm. Partial Differential Equations 40 (2015), 766-789.

6 V. A. Kondratiev and E. M. Landis, E.M. Qualitative Theory of Second Order Linear Partial Differential Equations. Itogi Nauki i Tekhniki: Sovremennye Problemy Mat.: Fundamental'nye Napravleniya 32, VINITI, Moscow, 1988, 99-218 (English transl. in Partial Differential Equations III, Encyclopedia of Mathematical Sciences vol. 32 (Berlin: Springer, 1991)).

$7 \quad$ P. A. Kuchment, Floquet Theory for Partial Differential Equations. Operator Theory: Advances and Applications, vol. 60 (Basel: Birkhäuser, 1993).

8 V. Z. Meshkov. Weighted differential inequalities and their application for estimating the rate of decrease at infinity of solutions of second-order elliptic equations. Trudy Mat. Inst. Steklov 190 (1989), 139-158 (English transl. Proc. Steklov Inst. Math. 190 No. 1 (1992) 145-166).

9 V. Z. Meshkov. On the Possible Rate of Decay at Infinity of Solutions of Second Order Partial Differential Equations. Mat. Sbornik 182 No. 3 (1991) 364-383 (English transl. Math. USSR Sbornik 72 No. 2 (1992) 343-361).

10 G. Pall. The distribution of integers represented by binary quadratic forms. Bull. Amer. Math. Soc. 49 (1943), 447-449.

11 M. Reed and B. Simon. Methods of Modern Mathematical Physics IV: Analysis of Operators (San Diego: Academic Press, 1979).

12 B. Simon. Schrödinger Semigroups. Bull. Amer. Math. Soc. 7 (1982), 447-526.

13 L. E. Thomas. Time dependent approach to scattering from impurities in a crystal. Comm. Math. Phys. 33 (1973), 335-343. 